\title{
Force Distributions in Dense Two-Dimensional Granular Systems
}

\author{
Farhang Radjai, ${ }^{1}$ Michel Jean, ${ }^{2}$ Jean-Jacques Moreau, ${ }^{2}$ and Stéphane Roux ${ }^{3}$ \\ ${ }^{1}$ HLRZ, Forschungszentrum, 52425 Jülich, Germany \\ ${ }^{2}$ LMGC, Université de Montpellier II, Place Eugène Bataillon, 34095 Montpellier Cedex 05, France \\ ${ }^{3}$ LPMMH-ESPCI, 10 rue Vauquelin, 75231 Paris Cedex 05, France
}

\begin{abstract}
Relying on contact dynamics simulations, we study the statistical distribution of contact forces inside a confined packing of circular rigid disks with solid friction. We find the following: (1) The number of normal and tangential forces lower than their respective mean value decays as a power law. (2) The number of normal and tangential forces higher than their respective mean value decays exponentially. (3) The ratio of friction to normal force is uniformly distributed and is uncorrelated with normal force. (4) When normalized with respect to their mean values, these distributions are independent of sample size and particle size distribution.
\end{abstract}

Despite the highly uniform density of a random packing of noncohesive particles, photoelastic visualizations provide a striking evidence of the heterogeneous distribution of contact forces on a scale definitely larger than the typical particle size [1-3]. A quantitative characterization of these distributions is relevant both to mechanical processing (compression, compaction, flow, grinding) and fundamental understanding (mesoscopic scales, instability thresholds) of granular media [4-7].

This Letter is concerned with a numerical study of this problem in confined two-dimensional packings at static equilibrium. We are interested in the statistical distributions $P_{N}$ and $P_{T}$ of normal forces and (absolute values of) friction forces $N$ and $T$, independently of contact orientations. We also study the distribution $P_{\eta}$ of the dimensionless variable $\eta=T / N$, which is a measure of friction "mobilization" within the Coulomb range $[0, \mu]$, where $\mu$ is the coefficient of friction between disks. Scaling with sample size and relation among the three distributions will be considered too.

Numerical results will be presented here for four samples of 500, 1200, 4025, and 1024 particles, referred to as samples A, B, C, and D, respectively. Particle radii are uniformly distributed between 3.8 and $7.5 \mathrm{~mm}$ in samples A and B, and between 1.5 and $7.5 \mathrm{~mm}$ in sample C. Sample D contains 192 particles of radius $1.6 \mathrm{~mm}, 320$ particles of radius $1.05 \mathrm{~mm}$, and 512 particles of radius $0.65 \mathrm{~mm}$. Particles are contained in a rectangular frame composed of one planar base, two immobile walls, and one horizontal plane (the lid) free to move vertically and on which a downward force of $6600 \mathrm{~N}$ is applied. The acceleration of gravity is set to zero in order to avoid force gradients in the sample. Particle-particle and particle-base coefficients of friction are 0.2 and 0.5 , respectively. All other coefficients of friction are zero.

For this investigation, we have relied on the contact dynamics (CD) approach to the dynamics of perfectly rigid particles with unilateral contacts. Since particles cannot interpenetrate, the allowed configurations of the system, characterized by a set of inequalities, define a region in the configuration space presenting a large number of edges and corners. Moreover, the basic Coulomb's law of friction, relevant to most of the granular media of interest, is a nonsmooth law in the sense that friction force and sliding velocity at a contact are not related together as a function. Finally, in the case of collisions velocity jumps occur, so that the evolution is not globally governed by differential equations in the classical sense.

The most commonly used algorithms are based on regularization schemes. In this way, impenetrability is approximated by a steep repulsive potential and Coulomb's law by a viscous friction law, to which smooth computational methods can be applied. The dominant feature of the CD method is that the conditions of perfect rigidity and exact Coulombian friction are implemented, with no resort to any regularization. At a given step of evolution, all kinematic constraints implied by lasting interparticular contacts and the possible rolling of some particles over others are simultaneously taken into account, together with the equations of dynamics, in order to determine all contact forces in the system. The method is thus able to deal properly with the nonlocal character of the momentum transfers-resulting from the perfect rigidity of particles in contact.

Detailed descriptions of the CD method can be found in the literature [8-10]. In relation with the present investigation, we would just like to underline the point that dynamics is an essential ingredient of this approach. It is well known that a granular system at static equilibrium is hyperstatic; i.e., for given boundary conditions there is a continuous set of possible contact forces. This is due both to the absence of an internal displacement field (because of perfect rigidity) and to the nonsmooth character of the friction law [11]. In the $\mathrm{CD}$ method, the force network at static equilibrium is determined through the dynamic processes from which it relaxed. In other words, as in real granular systems, the static values of forces are reached 
asymptotically as the kinematic energy of the system is dissipated in friction and collisions.

Of course, this does not mean that the statistical distribution of forces is necessarily dependent on the preparation process. The most probable force distribution may well result from the generic disorder of granular systems [3]. However, density is a major control parameter of the mechanical properties of granular materials, and only in the steady state, reached after enough shear-induced volume change, it acquires a rather well-defined value for a given confining pressure [12]. That is why we applied the same procedure to prepare all samples in the same state: Filling the box with particles under gravity, shearing by moving the base horizontally (dilation occurs then), stopping shear and applying the confining load on top of the sample, and, finally, setting the gravity to zero and allowing the system to relax to equilibrium under the load. Although the algorithm is quite efficient compared to other available techniques, the whole procedure requires hundreds of CPU hours on a fast Unix workstation (Sparc 20) for each sample.

Figure 1 shows the network of normal forces in sample D. One can observe both large contact-to-contact fluctuations and a subnetwork of "force chains" that seem to carry a significant portion of the applied external stress. Forces range from 0.003 to 1127 N, i.e., a range of 6 orders of magnitude, which clearly requires a scaling analysis. The mean normal force is $\langle N\rangle=249 \mathrm{~N}$ and more than $60 \%$ of contacts carry a force lower than the mean.

Figure 2 displays semilogarithmic plots of probability distributions $P_{N}$ of normal forces in the four samples. Forces are normalized with respect to their mean in each sample. The normalized distributions coincide over almost the whole range, and the data for forces larger than

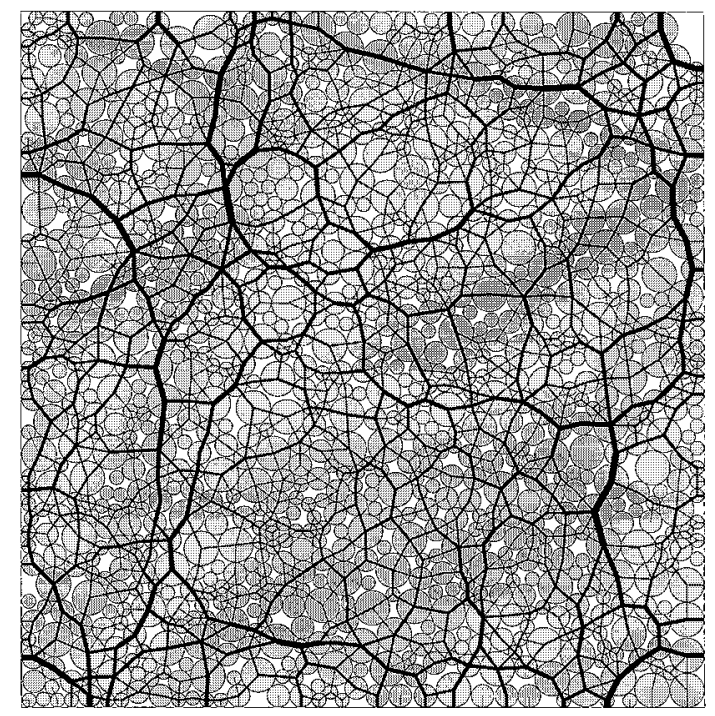

FIG. 1. Network of normal forces in sample D; see Table I. Forces are encoded as the widths of intercenter connecting segments. the mean are well fitted by an exponential decay. In order to see the behavior at low forces, we have shown in Fig. 3 the normalized log-log plots of the distribution of the logarithm of the forces. The data for forces lower than the mean have a power-law distribution. We conclude that the normalized distribution of normal forces is independent of our sample sizes and can be approximated by a power-law decay with a crossover to an exponential cutoff,

$$
P_{N} \propto \begin{cases}(N /\langle N\rangle)^{\alpha}, & N<\langle N\rangle, \\ e^{\beta(1-N /\langle N\rangle)}, & N>\langle N\rangle .\end{cases}
$$

We find $\alpha=-0.3$ and $\beta=1.4$. It is important to notice the collapse of normalized data on the same distribution in spite of the fact that the size dispersity of particles is not the same in all samples. The mean values seem, however, to depend on size dispersity since they do not scale with system size as shown in Table I. On the other hand, the lack of statistics at low normal forces in sample $\mathrm{D}$ as compared to sample $\mathrm{B}$, giving rise to the fluctuations observed in Fig. 3, suggests that the "branching process" generating low forces from the high applied force on the system is more efficient in systems with a continuous distribution of particle sizes.

The semilogarithmic and log-log plots of the probability distributions of the $T$ are displayed in Figs. 4 and 5. The data are normalized with respect to the mean $\langle T\rangle$ in each sample, and, as we see, they nicely collapse on the same distribution. This is again essentially a power-law decay with a crossover to an exponential cutoff,

$$
P_{T} \propto \begin{cases}(T /\langle T\rangle)^{\alpha^{\prime}} & T<\langle T\rangle, \\ e^{\beta^{\prime}(1-T /\langle T\rangle)}, & T>\langle T\rangle .\end{cases}
$$

We find $\alpha^{\prime}=-0.5$ and $\beta^{\prime}=1$.

We also studied the probability distribution $P_{\eta}$ of $\eta=$ $T / N$. This is a uniform distribution except for a small peak at $\eta=\mu$. The uniformity of this distribution may

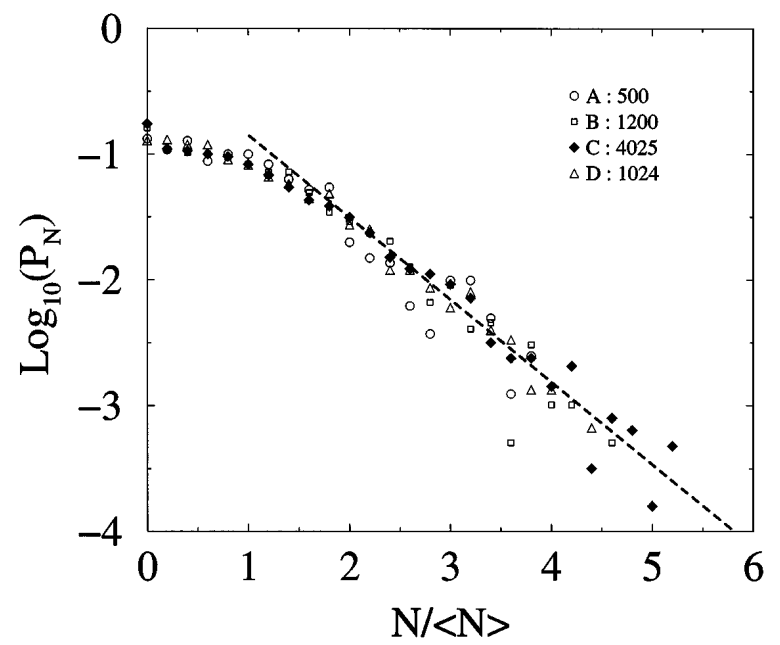

FIG. 2. Semilogarithmic plots of the probability distributions of normalized normal forces $N /\langle N\rangle$. 


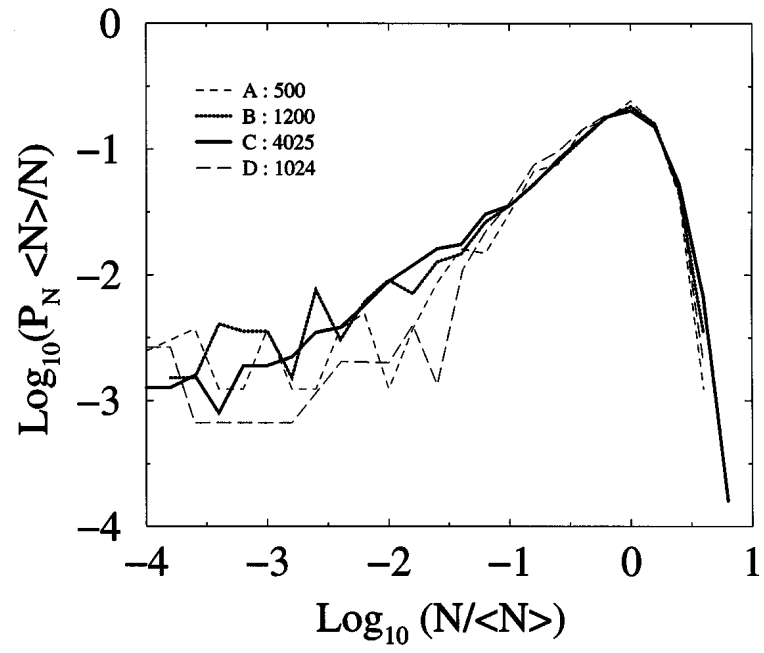

FIG. 3. Log-log plots of the probability distributions of normalized normal forces $N /\langle N\rangle$.

be attributed to the random structure of the contact network. On the other hand, it is likely that the rather weak singularity at $\eta=\mu$ is a "signature" of the dynamics of preparation. Indeed, only at sliding contacts is the friction force fully mobilized. If a granular assembly relaxes asymptotically towards static equilibrium, then the set of the last sliding contacts at the equilibrium threshold might remain fully mobilized. We checked that when the system is sheared by the motion of the basal plane, the peak at $\eta=\mu$ can rise to $50 \%$ of contacts, whereas the distribution remains uniform within $[0, \mu[$. Finally, we note that the normal forces for which $\eta=\mu$ are much smaller than the average, so that the peak may well result also from an imperfect relaxation.

Another important result regarding friction mobilization is the statistical independence of $\eta$ with respect to $N$. Whatever the value of $N$, friction is indifferently mobilized within the Coulomb range $[0, \mu N]$ (apart from the above discussed small peak). Such an assumption allows one to relate in a simple way $P_{N}$ to $P_{T}$. Let $P(N, T)$ be the joint probability distribution of normal and friction forces. Since $\eta$ is statistically independent of $N$, we may write $P(N, T)$ as a product of $P_{N}$ and $P_{\eta}$ times the Jacobian of the transformation $(N, T) \rightarrow(N, \eta)$,

$$
P(N, T) d N d T=\frac{1}{N} P_{N}(N) P_{\eta}(\eta) d N d T .
$$

TABLE I. Number of particles $p$, number of contacts $c$, width $L$, mean normal force $\langle N\rangle$, and mean friction force $\langle T\rangle$ in our samples A, B, C, and D.

\begin{tabular}{crrrcc}
\hline \hline Sample & \multicolumn{1}{c}{$p$} & \multicolumn{1}{c}{$c$} & $L(\mathrm{~mm})$ & $\langle N\rangle(N)$ & $\langle T\rangle(N)$ \\
\hline $\mathrm{A}$ & 500 & 806 & 260 & 592 & 51 \\
$\mathrm{~B}$ & 1200 & 1969 & 389 & 213 & 18 \\
$\mathrm{C}$ & 4025 & 6293 & 620 & 219 & 21 \\
$\mathrm{D}$ & 1024 & 1498 & 65 & 249 & 23 \\
\hline \hline
\end{tabular}

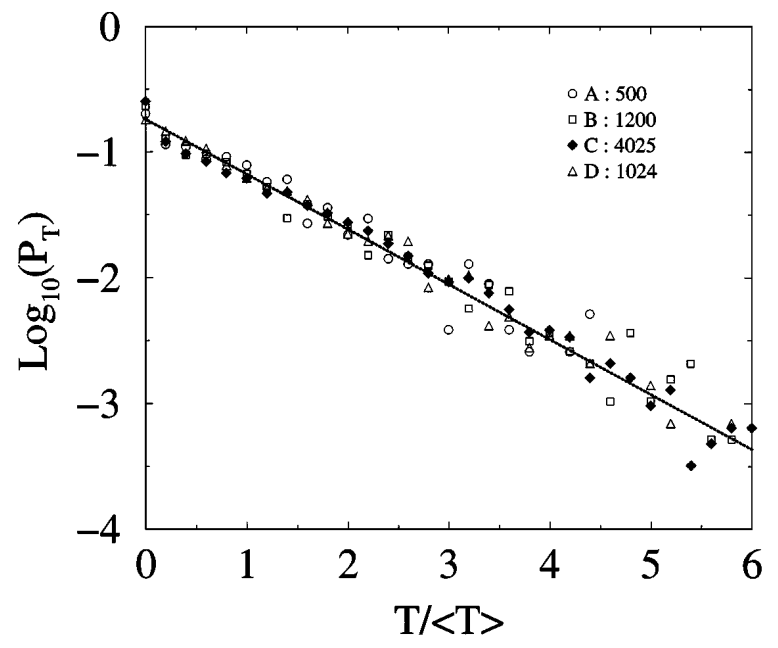

FIG. 4. Semilogarithmic plots of the probability distributions of normalized friction forces $T /\langle T\rangle$.

One may check that integration of the two members of Eq. (3) with respect to $N$ and $T$ over $[0,+\infty]$, with the substitution $T=\eta N$ in the right-hand side together with the constraint $\eta \in[0, \mu]$, implies a normalized $P_{\eta}$ over the Coulomb range $[0, \mu]$. Introducing the uniform distribution $P_{\eta}(\eta)=\mathbf{1}([0, \mu])$ in Eq. (3) and integrating with respect to $N$ over $[0,+\infty]$ with the substitution $N=T / \eta$ in the right side, we get the following relation between $P_{N}$ and $P_{T}$ :

$$
P_{T}(T)=\frac{1}{\mu} \int_{1 / \mu}^{+\infty} P_{N}(x T) \frac{d x}{x} .
$$

This equation implies that the initial power law of the two distributions $P_{N}$ and $P_{T}$ should be the same: $\alpha=$ $\alpha^{\prime}$. Moreover, an exponential upper cutoff of normal forces yields an exponential-integral cutoff for friction forces, i.e., essentially an exponential decay times a slowly varying function. Going back to Figs. 2-5, we see that such refinements are out of reach within the

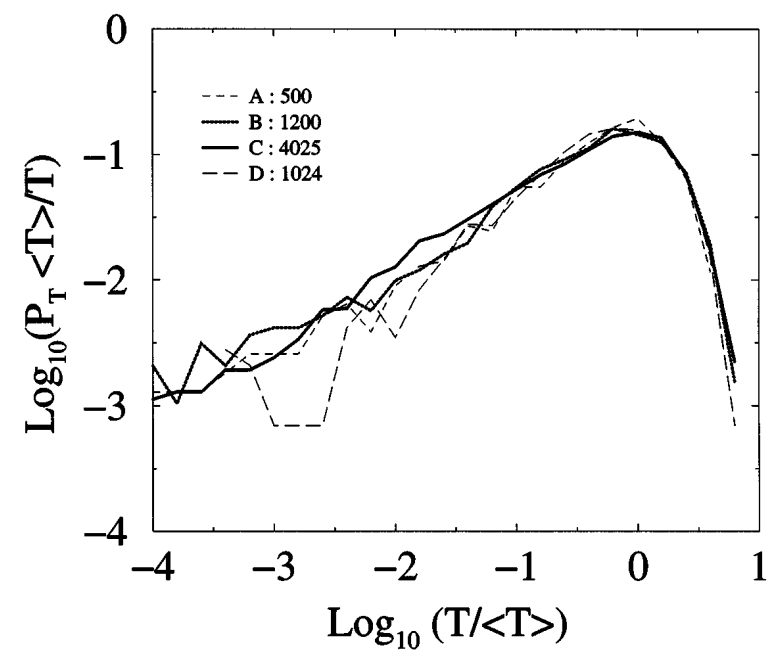

FIG. 5. Log-log plots of the probability distributions of normalized friction forces $T /\langle T\rangle$. 
statistical precision. On one hand, the cutoff may well be an exponential-integral function. On the other hand, the equality of exponents is consistent with the rough determination of these exponents.

Equation (4) can, however, be directly checked from the data. In Fig. 6 we have plotted both $P_{T}$ and the probability distribution obtained from $P_{N}$ via Eq. (4) for sample C. They are almost the same with a very good precision, although we assumed a uniform distribution of $\eta$ with no additional peak on the edge $\eta=\mu$. This validation of Eq. (4) is also an indirect check of the statistical independence of $\eta$ with respect to $N$.

Finally, integration of Eq. (4) with respect to $T$ yields the following relation between the mean values:

$$
\langle T\rangle=\frac{\mu}{2}\langle N\rangle .
$$

This relation is approximately satisfied for our samples, as can be seen in Table I.

In view of these findings, we would like to underline some salient aspects of the problem. One important point concerns the scale of statistical homogeneity of granular systems. Despite local force fluctuations, the present study shows that for a sample as small as 1200 particles the force distributions are clearly defined over several decades. An increase in sample size does nothing but improve statistics. Hence, as far as stress is concerned, the linear scale of statistical homogeneity in a 2D assembly is a few tens of particle diameters. This is what comes out also from the study of anisotropy in angular distributions of contact forces [13,14]. This observation is crucial for a continuum approach to the mechanics of granular media, needed in most of the usual technological problems.

Another point is that only the exponential tail of the distribution of normal forces, comprising nearly $40 \%$ of contacts in our simulations, has been observed in

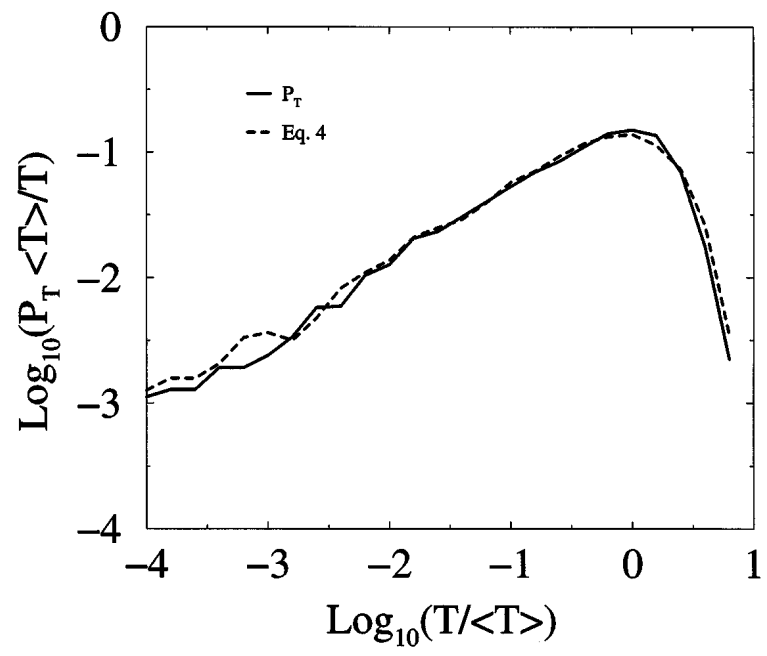

FIG. 6. Log-log plots of the distribution $P_{T}$ of normalized friction forces and the one obtained by applying Eq. (4) to $P_{N}$ in sample $\mathrm{C}$. experiments [3]. Weaker forces are technically difficult to measure, and their distribution has not been observed. The exponential tail has also been obtained through the usual simulation methods [15], and, what is more, a recent theoretical model provides plausible statistical arguments in favor of it [3]. This statistical model is likely to apply only to the subnetwork of force chains, which carries in effect most of the applied external load and in our simulations belongs to the exponential tail. The characteristic force at this scale is essentially imposed by the external load and the ratio of the system size to the largest particle size. On the other hand, the power-law decay of weak forces, if confirmed by other investigators, indicates the self-similar nature of weak contacts that do not belong to the subnetwork of large forces. Indeed, such contacts do not feel the external load, and hence their distribution can give rise to a power law through a self-similar branching process with no intrinsic scale. This observation also suggests that the exponents $\alpha$ and $\alpha^{\prime}$ depend on the interparticular friction coefficient $\mu$.

We gratefully acknowledge many fruitful conversations with D.E. Wolf. This work has been supported by the Groupement de Recherche "Physique des Milieux Hétérogènes Complexes" of the CNRS.

[1] P. Dantu, in Proceedings of the 4th International Conference on Soil Mechanics and Foundations Engineering (Butterworths Scientific Publications, London, 1957).

[2] T. Travers, D. Bideau, A. Gervois, and J.C. Messager, J. Phys. A 19, L1033-1038 (1986).

[3] C. H. Liu, S. R. Nagel, D. A. Schecter, S. N. Coppersmith, S. Majumdar, O. Narayan, and T. A. Witten, Science 269, 513 (1995).

[4] E. Guyon, S. Roux, A. Hansen, D. Bideau, J. P. Troadec, and H. Crapo, Rep. Prog. Phys. 53, 373-419 (1990).

[5] H. M. Jaeger and S. R. Nagel, Science 255, 1523 (1992).

[6] Disorder and Granular Media, edited by D. Bideau and A. Hansen (Elsevier, North-Holland, Amsterdam, 1993).

[7] D.E. Wolf, in Computational Physics: Selected Methods-Simple Exercises-Serious Applications, edited by K. H. Hoffmann and M. Schreiber (Springer, Heidelberg, 1996).

[8] J. J. Moreau, Eur. J. Mech. A, Solids 13, 93-114 (1994).

[9] M. Jean, in Mechanics of Geometrical Interfaces, edited by A. P. S. Selvadurai and M. J. Boulon (Elsevier Science B. V., Amsterdam, 1995), pp. 463-486.

[10] F. Radjai and S. Roux, Phys. Rev. E 51, 6177 (1995).

[11] F. Radjai, L. Brendel, and S. Roux, Phys. Rev. E (to be published).

[12] A. N. Schofield and C.P. Wroth, Critical State Soil Mechanics (McGraw-Hill, London, 1968).

[13] L. Rothenburg and R. J. Bathurst, Géotechnique 39, 601614 (1989).

[14] B. Cambou, in Powders and Grains 93, edited by C. Thornton (Balkema, Rotterdam, 1993).

[15] K. Bagi, in Powders and Grains 93 (Ref. [14]). 